\title{
Linearization and Analysis of Electrostatically Actuated MEMS 2D Optical Scanner
}

\author{
Contact: Hiroshi Toshiyoshi \\ 63-128 Engineering IV, Electrical Engineering, \\ UCLA, Los Angeles, CA 90095-1594 \\ hiroshi@ee.ucla.edu, fax: 310-794-5513
}

\author{
Hiroshi Toshiyoshi, Wibool Piyawattanametha, Cheng Ta Chan, and Ming C. Wu \\ Electrical Engineering, University of California, Los Angeles
}

MEMS 2D optical scanners have attracted much attention because of their applications in free-space fiber optic switching matrix [1], projection displays [2], confocal microscopes [3], free-space laser communications, and laser radars. Electrostatic actuation offers many advantages for scanners, including low power consumption, fast response time, and simple drive electronics. However, the electrostatic 2D scanners have highly nonlinear transfer characteristics, which makes control of the scanners difficult. In this paper, we present a linearization scheme that effectively suppress the nonlinearity of electrostatic MEMS 2D scanners. A theoretical model has been developed to predict the linearity of $2 \mathrm{D}$ scanner. The simulation results agree very well with experimental data.

Figure 1 shows the SEM of the 2D scanner used in our experiment. It is fabricated by surface-micromachining technique. The micromirror is suspended by two pairs of nested torsion bars ( $2 \mu \mathrm{m}$ wide, $200 \mu \mathrm{m}$ long, and 1.5 $\mu \mathrm{m}$ thick) for $\mathrm{X}$ - and $\mathrm{Y}$-scan. To achieve large scan angle, the micromirror (400 $\mu \mathrm{m} \times 400 \mu \mathrm{m})$ is suspended above the substrate with an air gap of $72 \mu \mathrm{m}$ using the self-assembled micro-elevator structure [4]. The scanning is controlled by quadrant electrodes undemeath the micromirror $\left(V_{1}, V_{2}, V_{3}, V_{4}\right)$.

The experimental set-up is shown in Table I(f). A position-sensitive detector (PSD) was used to measure the beam position as a function of the bias voltages. The procedure for calculating the projected beam pattem is outlined in Figure 2. Details of the model will be presented in the conference.

The experimental and calculated scan pattems are summarized in Table 1 for various driving scheme: Column1 shows the pattem obtained by simply superpositioning the driving voltages $V_{x}$ and $V_{y}$, as described in the table. Due to the large nonlinearity and voltage crosstalk of electrostatic operation, the image is skewed towards the comers by as much as $72 \%$ of the scan range (see Table I(a)). The linearization scheme we proposed is shown in Column-2: by pre-biasing the mirror with a $\mathrm{DC}$ voltage $\left(\mathrm{V}_{\text {bias }}\right)$ and applying differential voltages $\left(\mathrm{V}_{\mathrm{x}}+\mathrm{V}_{\mathrm{y}},-\mathrm{V}_{\mathrm{x}}+\mathrm{V}_{\mathrm{y}}, \quad-\mathrm{V}_{\mathrm{x}}-\mathrm{V}_{\mathrm{y}}, \mathrm{V}_{\mathrm{x}}-\mathrm{V}_{\mathrm{y}}\right)$ to the quadrant electrodes, the maximum distortion has been dramatically suppressed down to $13.3 \%$. The experimental data (Table I(b)) agrees very well with the simulation results (Table I(e)). Differential voltage operation has been known to improve the linearity of ID actuators such as a comb-drive mechanism or $1 \mathrm{D}$ torsion mirrors. To our knowledge, this is the first report on the linearization of $2 \mathrm{D}$ scanners.

We have also studied the effect of mirror shape on the linearity of 2D scanners. We have found that a circular mirror is expected to have the lowest distortion (1.5\%), compared to $13.3 \%$ for square mirrors. Perfect grid scanning pattem can be obtained by pre-distorting the driving voltages using the data obtained in Table I(b). Distortion as low as $0.33 \%$ has been achieved, as shown in Table I(c).

In summary, we have proposed and demonstrated a linearization scheme for electrostatic MEMS 2D optical scanners. We have also studied the effect of mirror shape on the linearity, and found that circular mirror has the lowest distortion.

[1] D. T. Neilson et al., "Fully Provisioned 112x112 Micro-Mechanical Optical Crossconnect with 35.8 Tb/s Demonstrated Capacity," OFC 2000, San Francisco, PD12-1

[2] M.-H Kiang et al., "Actuated Polysilicon Micromirrors for Raster-Scanning Displays," Transducers 97, Chicago.

[3] W. Piyawattanametha et al., "Surface-Micromachined Confocal Scanning Optical Microscope," CLEO 2000 (to be presented)

[4] L. Fan, et al., "Two-Dimensional Optical Scanner with Large Angular Rotation Realized by Self-Assembled Micro-Elevator," IEEE LEOS Summer Topical Meeting on Optical MEMS, 1998, Monterey.

500 words

(1) physical sensors, actuators, or systems 


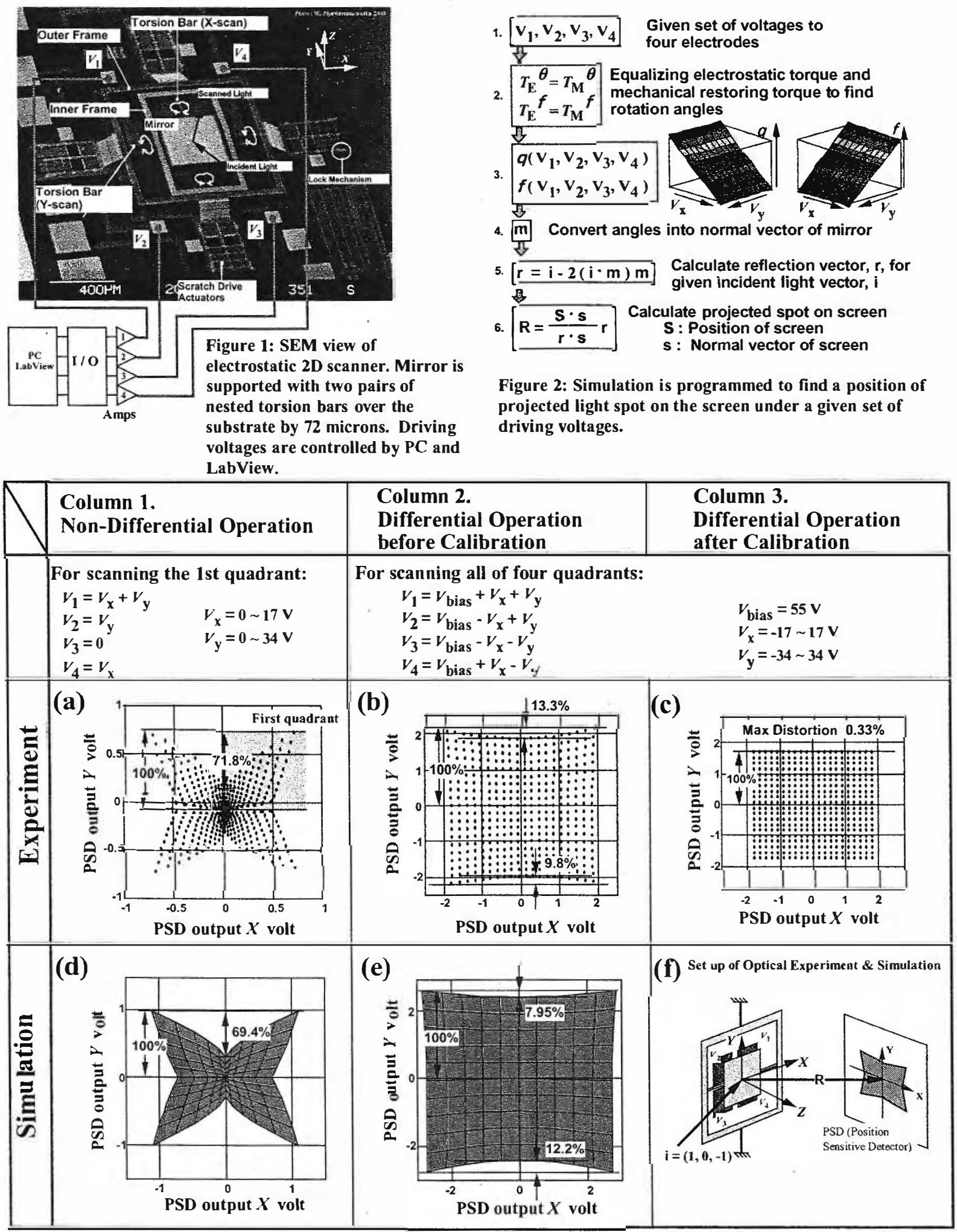

Table I: Experimental (upper row) and simulation (lower row) results of scanned grid patterns. As predicted by the simulation, differential voltage operation has been found to be essential for 2D scan of small image distortion. Regular grid patterns can be obtained after calibrating the voltage-angle characteristics. 